G. Cao

Nagoya Math. J.

Vol. 185 (2007), 171-186

\title{
TOEPLITZ ALGEBRAS ON STRONGLY PSEUDOCONVEX DOMAINS
}

\author{
GUANGFU CAO
}

\begin{abstract}
In the present paper, it is proved that the $K_{0}$-group of a Toeplitz algebra on any strongly pseudoconvex domain is always isomorphic to the $K_{0^{-}}$ group of the relative continuous function algebra, and is thus isomorphic to the topological $K^{0}$-group of the boundary of the relative domain. Further there exists a ring isomorphism between the $K_{0}$-groups of Toeplitz algebras and the Chern classes of the relative boundaries of strongly pseudoconvex domains. As applications of our main result, $K$-groups of Toeplitz algebras on some special strongly pseudoconvex domains are computed. Our results show that the Toeplitz algebras on strongly pseudoconvex domains have rich structures, which deeply depend on the topological structures of relative domains. In addition, the first cohomology groups of Toeplitz algebras are also computed.
\end{abstract}

\section{$\S 1$. Introduction}

Toeplitz operators $T_{\varphi}$ on the Hardy space $H^{2}(\mathbb{T})$ over the unit circle $\mathbb{T}$ are classically defined as follows;

$$
T_{\varphi} f=P(\varphi f), \quad \forall f \in H^{2}(\mathbb{T}),
$$

where $\varphi$ is a function in $L^{\infty}(\mathbb{T}), P$ is the orthogonal projection from $L^{2}(\mathbb{T})$ to $H^{2}(\mathbb{T})$ (cf. R. G. Douglas [1]). This definition can be generalized to any bounded domain. The study of the algebra generated by these operators is an important part of the theory of operator algebras. In particular, the extension theory of $C^{*}$-algebras has deep relations with the Toeplitz $C^{*}$ algebras.

The $K$-theory of the Toeplitz algebra plays an important role in the $K$-theory of operator algebras, beside the fact that it supplies important examples for general $K$-theory. However, the simplicity of the unit circle

Received April 3, 2003.

Revised September 21, 2004, March 31, 2005, March 3, 2006.

2000 Mathematics Subject Classification: 47B35.

Supported by National Natural Science Foundation of China. 
obscures the deeper relation between the analytical character of the Toeplitz algebra and the topological character of the underlying domain.

In this paper, we show how the $K_{0}$ of the domain and the boundary relate to each other. In fact, we obtain an isomorphism between the $K_{0^{-}}$ group of the Toeplitz algebra and the topological $K_{0}$-group of $\partial \Omega$ for any strongly pseudoconvex domain $\Omega$. That is

TheOREM 1. Suppose $\Omega$ is a strongly pseudoconvex domain, then

$$
K_{0}\left(\mathcal{J}_{\partial \Omega}\right) \cong K_{0}(C(\partial \Omega)) \cong K^{0}(\partial \Omega)
$$

where $K^{0}(\partial \Omega)$ denotes the topological $K^{0}$-group of $\partial \Omega$.

This shows that $K_{0}\left(\mathcal{J}_{\partial \Omega}\right)$ depends only on the topology of $\partial \Omega$. Using this result, we give some explicit computation of the $K$-groups of some Toeplitz algebras. Let us note that explicit computations have been known only for the case of the unit ball. Curto matrices are our main technical tool for the computation of $K$-theory. Via Chern characters, we see how the $K$-groups of Toeplitz algebras of a domain relate to the topology of the boundary. In order to see this, we first note that

$$
\operatorname{ch}: K_{0}(X) \longrightarrow H^{e v}(X ; \mathbb{Q}),
$$

is a ring homomorphism from the $K$-theory of $X$ into the even cohomology of $X$ with rational coefficients, and induces a ring isomorphism $K_{0}(X) \otimes \mathbb{Q} \rightarrow$ $H^{e v}(X ; \mathbb{Q})$ for any compact Hausdorff space $X$. Then we obtain a ring isomorphism between $K_{0}\left(\mathcal{J}_{\partial \Omega}\right) \otimes \mathbb{Q}$ and $H^{e v}(\partial \Omega ; \mathbb{Q}$ ) by Theorem 1 (see $[19])$.

Let us put our results in historical context. Since the 1970's, a list of specialists are interested in the Toeplitz operators and algebras on general domains in the complex plane and domains in the $n$-dimensional complex space $\mathbb{C}^{n}$. S. Axler, J. B. Conway and G. McDonald [2] studied the Toeplitz algebras on connected domains in the complex plane.

In [3] and [4], we studied the $K_{0}$-groups of Toeplitz algebras on connected domains. L. A. Coburn [5] studied Toeplitz operators on the unit ball in $\mathbb{C}^{n}$ and obtained the so-called Coburn exact sequence

Except for the unit disk in $\mathbb{C}$ and the unit ball in $\mathbb{C}^{n}$, a class of more general important domains are strongly pseudoconvex domains in $\mathbb{C}^{n}$. U. Venugopalkrishna [6] studied the Fredholm Toeplitz operators on these domains. 
In fact, there are a series of papers on Toeplitz operators on strongly pseudoconvex domains. For example, one may consult H. Upmeier [7], N. Salinas, A. Sheu, and H. Upmeier [8], N. P. Jewell and S. G. Krantz [9], I. Raeburn [10], N. P. Jewell [11], J. Janas [12], H. Sato and K. Yabuta [13] and K. Yabuta [14] etc. L. Boutet de Monvel and Guillemin [15], Guillemin [16] and L. Boutet de Monvel [17] studied Toeplitz operators on strongly pseudoconvex domains contained in a complex analytic manifold. But the definition of Toeplitz operators depends on the notion of pseudodifferential operators.

\section{$\S 2 . \quad K$-groups of Toepliz algebras}

Let $\rho: \mathbb{C}^{n} \rightarrow R$ be a $C^{2}$ function. It is said to be the defining function of the bounded domain $\Omega$ defined as follows:

$$
\Omega=\left\{z \in \mathbb{C}^{n} \mid \rho(z)<0\right\}
$$

if the gradient $d \rho(z) \neq 0$ at each boundary point. Recall that the domain is said to be strongly pseudoconvex if for all vectors $\left(\xi_{1}, \ldots, \xi_{n}\right)$ in the complex tangent space of $\partial \Omega$ the Levi form is positive definite. To wit if

$$
\sum_{j} \frac{\partial \rho}{\partial z_{j}} \xi_{j}=0
$$

then we have

$$
\sum_{i, j} \frac{\partial^{2} \rho}{\partial \bar{z}_{i} \partial z_{j}} \bar{\xi}_{i} \xi_{j}>0
$$

Strongly pseudoconvex domains form an important class of domains of holomorphy which have rich geometric structure. The basic example is the unit ball $B_{n}$. Another instance is the "solid torus" $\Omega_{1}$ in $\mathbb{C}^{2}$ (see Krantz $[18$, p. 110]), where

$$
\begin{gathered}
\Omega_{1}=\left\{z=\left(z_{1}, z_{2}\right) \in \mathbb{C}^{2}: \rho_{1}\left(z_{1}, z_{2}\right)<0\right\}, \\
\rho_{1}\left(z_{1}, z_{2}\right)=\left|z_{1}\right|^{2}+\left|z_{2}\right|^{2}+1-2\left|z_{1}\right|-r^{2} \quad(0<r<1) .
\end{gathered}
$$

Lemma 1. Let $\Omega$ be a strongly pseudoconvex domain in $\mathbb{C}^{n}$. Let $\varphi=$ $\left(\varphi_{1}, \ldots, \varphi_{m}\right)$ be an $m$-tuple in $L^{\infty}(\Omega)$ and let $\varphi^{\wedge}$ be the Curto matrix of $\varphi$ (consult R. Curto [19]). Then

(i) $\operatorname{det}\left(\varphi^{\wedge}\right)=\left(\sum_{i=1}^{m}\left|\varphi_{i}\right|^{2}\right)^{2^{m-2}}$, 
(ii) $\varphi^{\wedge^{*}} \varphi^{\wedge}=\varphi^{\wedge} \varphi^{\wedge^{*}}=\operatorname{diag}\left(\sum_{i=1}^{m}\left|\varphi_{i}\right|^{2}\right)$.

Proof. Follows directly from the definition.

Without loss of generality, we may assume that $0 \notin \partial \Omega$. Then we have Lemma 2. ([6], [7], [19], [20]) Let $\Omega$ be as in Lemma 1 , and $0 \notin \partial \Omega$, then

$$
\operatorname{Ind} T_{z^{\wedge}}=\operatorname{Ind} T_{z}=-1 \text {, }
$$

where $T_{z}=\left(T_{z_{1}}, \ldots, T_{z_{n}}\right)$ is the multiplication tuple on $H^{2}(\partial \Omega)$.

Lemma 3. ([6]) Let $\Omega$ be a strongly pseudoconvex domain, and let $\mathcal{J}_{\partial \Omega}$ denote the $C^{*}$-algebra

$$
\mathcal{J}_{\partial \Omega}=C^{*}\left(T_{\varphi}: \varphi \in C(\partial \Omega)\right)
$$

generated by all Toeplitz operators $T_{\varphi}$ with continuous symbol function $\varphi \in$ $C(\partial \Omega)$. Then $\mathcal{J}_{\partial \Omega}$ contains the ideal $\mathcal{K}$ of all compact operators on $H^{2}(\partial \Omega)$ as its commutator ideal, and there is an exact sequence

$$
0 \longrightarrow \mathcal{K} \longrightarrow \mathcal{J}_{\partial \Omega} \stackrel{\sigma}{\longrightarrow} C(\partial \Omega) \longrightarrow 0
$$

of $C^{*}$-algebras, where $\partial \Omega$ is the topological boundary of $\Omega$, and $\sigma$ is the "symbol homomorphism", uniquely determined by the condition $\sigma\left(T_{\varphi}\right)=\varphi$ for all $\varphi \in C(\partial \Omega)$.

TheOREm 1. Suppose $\Omega$ is a strongly pseudoconvex domain, then

$$
K_{0}\left(\mathcal{J}_{\partial \Omega}\right) \cong K_{0}(C(\partial \Omega)) \cong K^{0}(\partial \Omega)
$$

where $K^{0}(\partial \Omega)$ denotes the topological $K^{0}$-group of $\partial \Omega$.

Proof. First, we prove that the index map

$$
\delta_{1}: K_{1}(C(\partial \Omega)) \longrightarrow K_{0}(\mathcal{K})
$$

is a surjection. Without loss of generality, assume $0 \notin \partial \Omega$ and $m \in K_{0}(\mathcal{K})$. Set

$$
\varphi^{\wedge}=\frac{z^{\wedge}}{\left(\operatorname{det} z^{\wedge}\right)^{\frac{1}{2^{n-1}}}}
$$


then

$$
\begin{aligned}
\varphi^{\wedge^{*}} \varphi^{\wedge} & =\frac{1}{\left(\operatorname{det} z^{\wedge}\right)^{\frac{1}{2^{n-2}}}}\left(\begin{array}{cccc}
\sum_{i=1}^{n}\left|z_{i}\right|^{2} & 0 & \cdots & 0 \\
0 & \sum_{i=1}^{n}\left|z_{i}\right|^{2} & \cdots & 0 \\
\cdots & \cdots & \cdots & \cdots \\
0 & 0 & \cdots & \sum_{i=1}^{n}\left|z_{i}\right|^{2}
\end{array}\right) \\
& =\frac{1}{\sum_{i=1}^{n}\left|z_{i}\right|^{2}}\left(\begin{array}{cccc}
\sum_{i=1}^{n}\left|z_{i}\right|^{2} & 0 & \cdots & 0 \\
0 & \sum_{i=1}^{n}\left|z_{i}\right|^{2} & \cdots & 0 \\
\cdots & \cdots & \cdots & \cdots \\
0 & 0 & \cdots & \sum_{i=1}^{n}\left|z_{i}\right|^{2}
\end{array}\right)=I .
\end{aligned}
$$

Thus

$$
T_{\varphi^{\wedge}}^{*} T_{\varphi^{\wedge}}-I=T_{\varphi^{\wedge}}^{*} T_{\varphi^{\wedge}}-T_{\varphi^{\wedge}} \varphi^{\wedge} \in \mathcal{K} .
$$

Further, note that:

$$
T_{\varphi^{\wedge}}^{*} T_{\varphi^{\wedge}}=I+K, \quad K \in \mathcal{K} .
$$

Note $\varphi^{\wedge} \sim_{h} z^{\wedge}$, in fact,

$$
\psi_{t}=\frac{z^{\wedge}}{t\left(\operatorname{det} z^{\wedge}\right)^{\frac{1}{2^{n-1}}}+(1-t)}
$$

is a homotopy between $\varphi^{\wedge}$ and $z^{\wedge}$. Since $\operatorname{Ind} T_{z^{\wedge}}=\operatorname{Ind} T_{z}=-1$, we see that Ind $T_{\varphi^{\wedge}}=-1$. Let $\varphi_{m}=\varphi^{\wedge * m}$, then $\varphi_{m} \in \mathcal{U}_{2^{n-1}}(C(\partial \Omega))$, and Ind $T_{\varphi_{m}}=m$. Let $T_{\varphi_{m}}=U_{m}\left|T_{\varphi_{m}}\right|$ be the polar decomposition of $T_{\varphi_{m}}$. Then since $\sigma$ is a homomorphism, $T_{\varphi_{m}}^{*} T_{\varphi_{m}}-T_{\left|\varphi_{m}\right|^{2}} \in M_{2^{n-1}}(\mathcal{K})$. Taking into account the fact that $\left|\varphi_{m}\right|=I$, we see that

$$
\left|T_{\varphi_{m}}\right|^{2}=T_{\varphi_{m}}^{*} T_{\varphi_{m}}=I+K \in M_{2^{n-1}}\left(\mathcal{J}_{\partial \Omega}\right),
$$

where $K \in M_{2^{n-1}}(\mathcal{K})$. Since $I+K \geq 0$, we see that $\sqrt{I+K}+I \geq I$. Thus $\sqrt{I+K}+I$ is invertible, further

$$
\sqrt{I+K}-I=K(\sqrt{I+K}+I)^{-1} \in M_{2^{n-1}}(\mathcal{K}) .
$$

This implies that

$$
\left|T_{\varphi_{m}}\right|=\sqrt{I+K}=I+(\sqrt{I+K}-I) \in M_{2^{n-1}}\left(\mathcal{J}_{\partial \Omega}\right) .
$$

Note

$$
T_{\varphi_{m}}=U_{m}\left|T_{\varphi_{m}}\right|=U_{m}+U_{m}(\sqrt{I+K}-I)
$$


and $U_{m}(\sqrt{I+K}-I) \in M_{2^{n-1}}(\mathcal{K})$, we have

$$
U_{m}=T_{\varphi_{m}}-U_{m}(\sqrt{I+K}-I) \in M_{m}\left(\mathcal{J}_{\partial \Omega}\right) .
$$

Thus $\left[U_{m}\right]=\left[T_{\varphi_{m}}\right]$ in $M_{2^{n-1}}\left(\mathcal{J}_{\partial \Omega} / \mathcal{K}\right)$. It is clear that Ind $U_{m}=m$. Since $U_{m}$ is a partial isometry, we see that both $U_{m}^{*} U_{m}=p$ and $U_{m} U_{m}^{*}=q$ are orthogonal projections such that

$$
\operatorname{Ker} p=\operatorname{Ker} U_{m}=\operatorname{Ker} T_{\varphi_{m}}=\operatorname{Rang}(I-p),
$$

and

$$
\operatorname{Ker} q=\operatorname{Ker} U_{m}^{*}=\left[\operatorname{Rang} U_{m}\right]^{\perp}=\left[\operatorname{Rang} T_{\varphi_{m}}\right]^{\perp}=\operatorname{Rang}(I-q) .
$$

This shows that $I-p, I-q \in M_{2^{n-1}}(\mathcal{F}) \subset M_{2^{n-1}}(\mathcal{K})$, where $\mathcal{F}$ is the set of finite rank operators. Hence

$$
\delta_{1}\left(\left[\varphi_{n}\right]_{1}\right)=[I-p]_{0}-[I-q]_{0}=m
$$

by the definition of the index map.

By Lemma 3, we obtain the six-term exact sequence

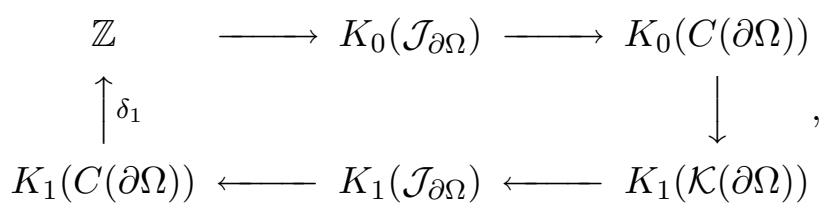

Clearly, $i^{*}: \mathbb{Z} \rightarrow K_{0}\left(\mathcal{J}_{\partial \Omega}\right)$ is a zero map since $\delta_{1}$ is surjective. Consequently, we obtain an exact sequence

$$
0 \longrightarrow K_{0}\left(\mathcal{J}_{\partial \Omega}\right) \stackrel{\rho^{*}}{\longrightarrow} K_{0}(C(\partial \Omega)) \longrightarrow 0
$$

Hence

$$
K_{0}\left(\mathcal{J}_{\partial \Omega}\right) \cong K_{0}(C(\partial \Omega))
$$

Since $K_{0}(C(X)) \cong K^{0}(X)$ as Abelian groups for any compact Hausdorff space $X$ (consult [22]), the proof of our theorem is thus complete.

TheOrem 2. Suppose $\Omega$ is a strongly pseudoconvex domain, then

$$
K_{1}(C(\partial \Omega)) \cong K_{1}\left(\mathcal{J}_{\partial \Omega}\right) \oplus \mathbb{Z}
$$


Proof. Since $K_{0}(\mathcal{K}(\partial \Omega)) \cong \mathbb{Z}, K_{1}(\mathcal{K}(\partial \Omega))=0$, by the six-term exact sequence

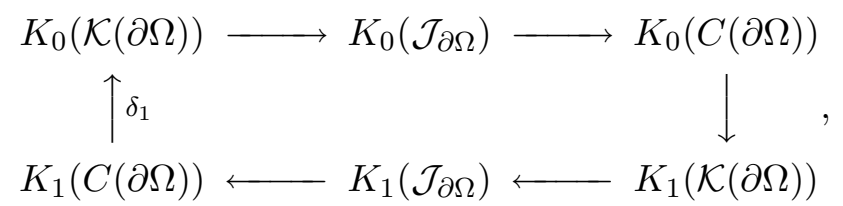

and the proof of Theorem 1, we obtain the following short exact sequence

$$
0 \longrightarrow K_{1}\left(\mathcal{J}_{\partial \Omega}\right) \longrightarrow K_{1}(C(\partial \Omega)) \stackrel{\delta_{1}}{\longrightarrow} \mathbb{Z} \longrightarrow 0 .
$$

Note $\mathbb{Z}$ is a free group with single generator, we see that

$$
K_{1}(C(\partial \Omega)) \cong K_{1}\left(\mathcal{J}_{\partial \Omega}\right) \oplus \mathbb{Z} .
$$

Remark. Theorem 1 shows that the $K_{0}$-theory of a Toeplitz algebra depends on the topological properties of the boundary of the underlying domain. Topological $K$-theory is related to the cohomology groups of the space in question via the Chern character, that is

Proposition 1. Suppose $X$ is a compact Hausdorff space, then the Chern character

$$
\operatorname{ch}: K_{0}(X) \longrightarrow H^{e v}(X ; \mathbb{Q}),
$$

is a ring homomorphism from the $K$-theory of $X$ into the even cohomology of $X$ with rational coefficients, and induces a ring isomorphism $K_{0}(X) \otimes \mathbb{Q} \rightarrow$ $H^{e v}(X ; \mathbb{Q})$.

Thus we obtain a ring isomorphism between $K_{0}\left(\mathcal{J}_{\partial \Omega}\right) \otimes \mathbb{Q}$ and $H^{e v}(\partial \Omega ; \mathbb{Q})$ by Theorem 1 and by using the Chern character (see [22]).

Proposition 2. If $\Omega=B_{n}$, the unit ball in $\mathbb{C}^{n}$, then the index map is an isomorphism.

Proof. In fact, if $\delta_{1}\left([\varphi]_{1}\right)=0$, without loss of generality, assume $\varphi \in$ $\mathcal{U}_{N}(C(\partial \Omega))$, then by the proof of the main theorem, we have Index $T_{\varphi}=0$, thus degree $\varphi=0$. By Hopf theorem (cf. J. Dugundji [21]), we know that $\varphi \sim_{h} I_{N}$, where $I_{N}$ is the unit in $\mathcal{U}_{N}(C(\partial \Omega))$. Thus $[\varphi]_{1}=0$. This shows that $\delta_{1}$ is an injective. Hence $\delta_{1}$ is indeed an isomorphism. 
Since the following sequence

$$
0 \longrightarrow K_{1}\left(\mathcal{J}_{\partial B_{n}}\right) \longrightarrow K_{1}\left(C\left(\partial B_{n}\right)\right) \stackrel{\delta_{1}}{\longrightarrow} \mathbb{Z} \longrightarrow 0
$$

is exact, we see that $K_{1}\left(\mathcal{J}_{\partial B_{n}}\right)=0$. On the other hand, since $K_{1}\left(C\left(\partial B_{n}\right)\right)=$ $\mathbb{Z}$, we have

Proposition 3. $K_{0}\left(\mathcal{J}_{\partial B_{n}}\right) \cong K_{0}\left(C\left(\partial B_{n}\right)\right) \cong \mathbb{Z}$

If $\partial \Omega$ is homotopically equivalent to $\partial B_{n}$, then the following statements still hold

(1) $\delta_{1}$ is an isomorphism,

(2) $K_{0}\left(\mathcal{J}_{\partial \Omega}\right)=\mathbb{Z}$

(3) $K_{1}\left(\mathcal{J}_{\partial \Omega}\right)=0$.

However, for a general domain $\Omega$, two elements in $\mathcal{U}_{\infty}(C(\partial \Omega))$ which have the same topological index may not be homotopic, hence $\delta_{1}$ may not be injective. Furthermore, by the exact sequence

$$
0 \longrightarrow K_{1}\left(\mathcal{J}_{\partial \Omega}\right) \longrightarrow K_{1}(C(\partial \Omega)) \stackrel{\delta_{1}}{\longrightarrow} \mathbb{Z} \longrightarrow 0,
$$

we know that $K_{1}\left(\mathcal{J}_{\partial \Omega}\right)$ can not be zero. For example, in [2], [3], we have proven that if $\Omega$ is a finite connected domain in the complex plane, then $K_{1}(C(\partial \Omega)) \cong \mathbb{Z}^{k}$, where $k$ is the number of holes in $\Omega$. Thus it is easy to see that $K_{1}(\mathcal{J}(\Omega))$ does not equal $\{0\}$ when $k>1$. In the following propositions, we will also see that for general strongly pseudoconvex domains, the $K$-groups of Toeplitz algebras depend on the domains.

Proposition 4. Suppose $\Omega \subset \mathbb{C}^{2}$ is the "solid torus" which is defined by defining function

$$
\rho\left(z_{1}, z_{2}\right)=\left(\left|z_{1}\right|-1\right)^{2}+\left|z_{2}\right|^{2}-r^{2} \quad(0<r<1),
$$

then

$$
K_{0}\left(\mathcal{J}_{\partial \Omega}\right) \cong K_{0}(C(\partial \Omega)) \cong \mathbb{Z} \oplus \mathbb{Z}
$$

and

$$
K_{1}\left(\mathcal{J}_{\partial \Omega}\right) \neq 0 .
$$


Proof. Write

$$
G=\left\{\left(\frac{z_{1}}{\left|z_{1}\right|},\left|z_{1}\right|, z_{2}\right) \mid\left(z_{1}, z_{2}\right) \in \partial \Omega\right\},
$$

then $G$ is homeomorphic to $\partial \Omega$. It is obvious that

$$
G \stackrel{\text { homeo }}{\cong} \mathbb{T} \times S_{r}^{2},
$$

where $S_{r}^{2}$ is the sphere with radius $r$ and center $(1,0,0)$ in $\mathbb{R}^{3}$. Thus

$$
C(\partial \Omega) \cong C\left(\mathbb{T} \times S_{r}^{2}\right) \cong C\left(\mathbb{T}, C\left(S_{r}^{2}\right)\right)=\mathbb{T}\left(C\left(S_{r}^{2}\right)\right) .
$$

Since the sequence

$$
0 \longrightarrow S C\left(S_{r}^{2}\right) \longrightarrow \mathbb{T}\left(C\left(S_{r}^{2}\right)\right) \longrightarrow C\left(S_{r}^{2}\right) \longrightarrow 0
$$

is split exact, where $S C\left(S_{r}^{2}\right)$ is the suspension of $C\left(S_{r}^{2}\right)$ (see [22]), we see that

$$
K_{i}(C(\partial \Omega)) \cong K_{i}\left(\mathbb{T}\left(C\left(S_{r}^{2}\right)\right)\right) \cong K_{i}\left(C\left(S_{r}^{2}\right)\right) \oplus K_{i+1}\left(C\left(S_{r}^{2}\right)\right), \quad(i=1,2) .
$$

Thus

$$
\begin{aligned}
& K_{0}(C(\partial \Omega)) \cong K_{0}\left(C\left(S_{r}^{2}\right)\right) \oplus K_{1}\left(C\left(S_{r}^{2}\right)\right) \cong \mathbb{Z} \oplus \mathbb{Z}, \\
& K_{1}(C(\partial \Omega)) \cong K_{0}\left(C\left(S_{r}^{2}\right)\right) \oplus K_{2}\left(C\left(S_{r}^{2}\right)\right) \cong \mathbb{Z} \oplus \mathbb{Z} .
\end{aligned}
$$

Hence

$$
K_{0}\left(\mathcal{J}_{\partial \Omega}\right)=\mathbb{Z} \oplus \mathbb{Z}
$$

By Theorem 2, we see that $K_{1}\left(\mathcal{J}_{\partial \Omega}\right) \neq 0$.

Remark. We know that for $0<r<1 / 2$, the function

$$
\rho\left(z_{1}, z_{2}\right)=\left|z_{1}\right|^{2}+\left|z_{2}\right|^{2}+1-2 \sqrt{x_{1}^{2}+x_{2}^{2}}-r^{2}
$$

defines a strongly pseudoconvex domain

$$
\Omega=\left\{\left(z_{1}, z_{2}\right) \mid \rho\left(z_{1}, z_{2}\right)<0\right\}
$$

where $z_{1}=x_{1}+i y_{1}, z_{2}=x_{2}+i y_{2}$. Set

$$
\varphi\left(z_{1}, z_{2}\right)=\left(\frac{z_{1}+\overline{z_{1}}}{2}+i \frac{z_{2}+\overline{z_{2}}}{2}, \frac{z_{1}-\overline{z_{1}}}{2 i}+i \frac{z_{2}-\overline{z_{2}}}{2 i}\right),
$$


then $\varphi$ is a homeomorphism from $\partial \Omega$ onto

$$
G=\left\{\left.\left(w_{1}, w_{2}\right)\left|\left(\left|w_{1}\right|-1\right)^{2}+\right| w_{2}\right|^{2}=r^{2}\right\},
$$

where $\left(w_{1}, w_{2}\right)=\varphi\left(z_{1}, z_{2}\right)$ for arbitrary $\left(z_{1}, z_{2}\right) \in \partial \Omega$. Thus, we have

$$
K_{0}\left(\mathcal{J}_{\partial \Omega}\right) \cong K_{0}(C(\partial \Omega)) \cong K_{0}(C(G)) \cong \mathbb{Z} \oplus \mathbb{Z}
$$

Now let us consider another strongly pseudoconvex domain. Write

$$
\Omega=\left\{\left(z_{1}, z_{2}\right) \mid\left(\left|z_{1}\right|-1\right)^{2}+\left(\left|z_{2}\right|-1\right)^{2}<r^{2}\right\} \quad(0<r<1 / 2),
$$

then $\Omega$ is a strongly pseudoconvex domain, and

$$
\partial \Omega=\left\{\left(z_{1}, z_{2}\right) \mid\left(\left|z_{1}\right|-1\right)^{2}+\left(\left|z_{2}\right|-1\right)^{2}=r^{2}\right\} .
$$

We have the following

Proposition 5. Suppose $\Omega \subset \mathbb{C}^{2}$ is the strongly pseudoconvex domain which is defined by defining function

$$
\rho\left(z_{1}, z_{2}\right)=\left(\left|z_{1}\right|-1\right)^{2}+\left(\left|z_{2}\right|-1\right)^{2}-r^{2} \quad(0<r<1 / 2),
$$

then

$$
K_{0}\left(\mathcal{J}_{\partial \Omega}\right) \cong K_{0}(C(\partial \Omega)) \cong \mathbb{Z}^{4}
$$

and

$$
K_{1}\left(\mathcal{J}_{\partial \Omega}\right) \neq 0
$$

Proof. Write

$$
G=\left\{\left(\frac{z_{1}}{\left|z_{1}\right|}, \frac{z_{2}}{\left|z_{2}\right|},\left|z_{1}\right|,\left|z_{2}\right|\right) \mid\left(z_{1}, z_{2}\right) \in \partial \Omega\right\},
$$

then $G$ is homeomorphic to $\partial \Omega$, thus

$$
C(\partial \Omega) \cong C(G)
$$

It is obvious to see that

$$
G \stackrel{\text { homeo }}{\cong} \mathbb{T} \times \mathbb{T} \times \mathbb{T}_{r},
$$

where $\mathbb{T}_{r}$ is the circle with radius $r$ and center $(1,1)$. Hence

$$
C(G) \cong C\left(\mathbb{T} \times \mathbb{T} \times \mathbb{T}_{r}\right) \cong C\left(\mathbb{T}^{3}\right) .
$$


Further,

$$
K_{i}(C(G)) \cong \mathbb{Z}^{4}, \quad(i=1,2)
$$

This follows that

$$
K_{0}\left(\mathcal{J}_{\partial \Omega}\right) \cong K_{0}(C(\partial \Omega)) \cong K_{0}(C(G)) \cong \mathbb{Z}^{4} .
$$

Further $K_{1}\left(\mathcal{J}_{\partial \Omega}\right) \neq 0$ by Theorem 2 .

In general, we may define function $\rho$ in $\mathbb{C}^{n}$ as

$$
\rho\left(z_{1}, \ldots, z_{n}\right)=\sum_{i=1}^{n}\left(\left|z_{i}\right|-1\right)^{2}-r^{2}, \quad(0<r<1 / 2) .
$$

Then

$$
\Omega=\left\{\left(z_{1}, \ldots, z_{n}\right) \mid \rho\left(z_{1}, \ldots, z_{n}\right)<0\right\}
$$

is a strongly pseudoconvex domain in $\mathbb{C}^{n}$. Similar to Proposition 5 , we have the following

Proposition 6. Suppose $\Omega \subset \mathbb{C}^{n}$ is the strongly pseudoconvex domain which is defined by defining function

$$
\rho\left(z_{1}, \ldots, z_{n}\right)=\sum_{i=1}^{n}\left(\left|z_{i}\right|-1\right)^{2}-r^{2}, \quad(0<r<1 / 2)
$$

then

$$
K_{0}\left(\mathcal{J}_{\partial \Omega}\right) \cong K_{0}(C(\partial \Omega)) \cong \mathbb{Z}^{2^{n}}
$$

and

$$
K_{1}\left(\mathcal{J}_{\partial \Omega}\right) \neq 0 .
$$

Proof. Write

$$
G=\left\{\left(\frac{z_{1}}{\left|z_{1}\right|}, \ldots, \frac{z_{n}}{\left|z_{2}\right|},\left|z_{1}\right|, \ldots,\left|z_{n}\right|\right) \mid\left(z_{1}, \ldots, z_{n}\right) \in \partial \Omega\right\},
$$

then

$$
\partial \Omega \stackrel{\text { homeo }}{\cong} G \stackrel{\text { homeo }}{\cong} \mathbb{T}^{n} \times S_{r}^{n-1},
$$

where $S_{r}^{n-1}$ is the sphere with radius $r$ and center $(1, \ldots, 1)$ in $\mathbb{R}^{n}$. Similar to the proof of Proposition 1, we can easily prove that

$$
K_{i}(C(\partial \Omega)) \cong\left[K_{i}\left(C\left(S_{r}^{n-1}\right)\right)\right]^{2^{n-1}} \oplus\left[K_{i+1}\left(C\left(S_{r}^{n-1}\right)\right)\right]^{2^{n-1}} \cong \mathbb{Z}^{2^{n}} \quad(i=1,2)
$$


by induction. Hence

$$
K_{0}\left(\mathcal{J}_{\partial \Omega}\right) \cong \mathbb{Z}^{2^{n}}
$$

and

$$
K_{1}\left(\mathcal{J}_{\partial \Omega}\right) \neq 0 .
$$

We are done.

We know that for any compact Hausdorff space $X, K_{1}(C(X))$ is related to the cohomotopy group $\pi^{1}(X)$ of $X$. Recall that

$$
\pi^{1}(X)=\{[f] \mid f \in C(X, \mathbb{T})\}
$$

where $[f]$ denotes the homotopy equivalence class of $f$.

In fact, there is a homomorphism $\Delta$ from $K_{1}(C(X))$ to $\pi^{1}(X)$ such that the following sequence

$$
0 \longrightarrow \operatorname{Ker} \Delta \longrightarrow K_{1}(C(X)) \longrightarrow \pi^{1}(X) \longrightarrow 0
$$

is split exact. In general, $\operatorname{Ker} \Delta$ may be nontrivial, for example, if $k>1$, then $\pi^{1}\left(S^{k}\right)=0$, however, $K_{1}\left(C\left(S^{k}\right)\right) \cong \mathbb{Z}$ for any odd number $k>1$.

We state the following problem for future reference.

Problem. For which compact Hausdorff space $X$, we have

$$
K_{1}(C(X)) \cong \pi^{1}(X) ?
$$

If $X \subset \mathbb{C}$, it is well-known that $K^{1}(X)=H^{1}(X, \mathbb{Z})=\pi^{1}(X)$, where $K^{1}(X)$ is the topological $K^{1}$-group of $X$, and $H^{1}(X, \mathbb{Z})$ is the first Cech cohomological group of $X$ (consult R. G. Douglas [23]). In [3], we proved that if $X$ is a finitely connected domain in $\mathbb{C}$, then $K_{1}(C(\partial X))=\pi^{1}(\partial X)$. In [4], we obtained an analogue of the result for some special infinitely connected domains. It seems that the above problem has an affirmative answer for the boundaries of connected domains in the complex plane.

\section{§3. Cohomology groups of Toeplitz algebras}

The cohomology group of an operator algebra is an important concept, where the first cohomology group $H^{1}$ is deeply relative to the derivation. Recall a linear derivation on an algebra $\mathcal{B}$ is a linear map $\delta$ from $\mathcal{B}$ into $\mathcal{B}$ which satisfies that $\delta(f g)=\delta(f) g+f \delta(g)$. Sakai's theorem says that each linear derivation on $C^{*}$-algebra is continuous (see [24]). If there is a 
$f \in \mathcal{B}$ such that $\delta(g)=f g-g f$ for any $g \in \mathcal{B}$, then $\delta$ is said to be an inner derivation on $\mathcal{B}$, otherwise, $\delta$ is called an outer derivation. Ringrose's deep result indicates that there are only inner derivations on any Von-Neumann algebras. In the case of $C^{*}$-algebras, there may be many outer derivations, for instance, each bounded linear operator (on a Hilbert space $H$ ) induced a derivation on the compact operator ideal, hence the first cohomology group of $\mathcal{K}$ is $L(H) / \mathcal{K}$. In this section, we will compute the first cohomology group of $\mathcal{J}_{\partial \Omega}$.

Proposition 7. Suppose $\Omega$ is a strongly pseudoconvex domain in $\mathbb{C}^{n}$, and $\delta$ is a linear derivation on $\mathcal{J}_{\partial \Omega}$, then the range of $\delta$ is contained in $\mathcal{K}$.

Proof. Since the convex combination of unitary elements in $\mathcal{J}_{\partial \Omega}$ is dense in the unit ball of $\mathcal{J}_{\partial \Omega}$ (see [25]), we need only to prove that $\delta(U) \in \mathcal{K}$ for any unitary operator $U$ in $\mathcal{J}_{\partial \Omega}$. Assume $U \in \mathcal{J}_{\partial \Omega}$ is a unitary operator, then there is a $\varphi_{U} \in C(\partial \Omega)$ such that $\xi([U])=\varphi_{U}$. By $U^{*} U=I$, we have $\left|\varphi_{U}\right|^{2}=\overline{\varphi_{U}} \varphi_{U}=\xi\left(\left[U^{*}\right]\right) \xi([U])=\xi([I])=1$, this shows that $\varphi_{U}$ is a unimodular function. Write $\delta(U)=T_{U}$, then there is a $\varphi_{T_{U}} \in C(\partial \Omega)$ such that $\xi\left(\left[T_{U}\right]\right)=\varphi_{T_{U}}$. Note that all elements in $\mathcal{J}_{\partial \Omega}$ are essentially commutative, we see that $\left[\delta\left(U^{m}\right)\right]=m\left[U^{m-1} \delta(U)\right]$, hence $\xi\left(\left[\delta\left(U^{m}\right)\right]\right)=$ $m \xi\left(\left[U^{m-1}\right]\right) \xi([\delta(U)])=m \varphi_{U}^{m-1} \varphi_{T_{U}}$, further $\left\|\xi\left(\left[\delta\left(U^{m}\right)\right]\right)\right\|_{\infty}=m\left\|\varphi_{T_{U}}\right\|_{\infty}$ since $\left|\varphi_{U}\right|=1$. By $\left\|U^{m}\right\|=1$, we have $\left\|\xi\left(\left[\delta\left(U^{m}\right)\right]\right)\right\|=\left\|\left[\delta\left(U^{m}\right)\right]\right\| \leq$ $\left\|\delta\left(U^{m}\right)\right\| \leq\|\delta\|\left\|U^{m}\right\|=\|\delta\|<\infty$ for any $m \in \mathbb{N}$, this follows that $\varphi_{T_{U}}=0$, so that $\delta(U)=T_{U} \in \mathcal{K}$ (since $\xi$ is an isometrical isomorphism from $\mathcal{J}_{\partial \Omega} / \mathcal{K}$ onto $C(\partial \Omega))$. We are done.

Let $B\left(\mathcal{J}_{\partial \Omega}, \mathcal{J}_{\partial \Omega}\right)$ be the set of all derivations from $\mathcal{J}_{\partial \Omega}$ into itself, and $Z\left(\mathcal{J}_{\partial \Omega}, \mathcal{J}_{\partial \Omega}\right)$ be the set of all inner derivations on $\mathcal{J}_{\partial \Omega} . H^{1}\left(\mathcal{J}_{\partial \Omega}, \mathcal{J}_{\partial \Omega}\right)=$ $B\left(\mathcal{J}_{\partial \Omega}, \mathcal{J}_{\partial \Omega}\right) / Z\left(\mathcal{J}_{\partial \Omega}, \mathcal{J}_{\partial \Omega}\right)$ is said to be the first cohomology group of $\mathcal{J}_{\partial \Omega}$ (The definition on higher order cohomology groups of Banach algebras can be find in $[26])$.

THEOREM 3. For any strongly pseudoconvex domain $\Omega$ in $\mathbb{C}^{n}$, we have

$$
H^{1}\left(\mathcal{J}_{\partial \Omega}, \mathcal{J}_{\partial \Omega}\right) \cong\left\{T_{z_{1}}, \ldots, T_{z_{n}}\right\}_{e}^{\prime} / \mathcal{J}_{\partial \Omega} .
$$

Proof. For any $f, g \in \mathcal{J}_{\partial \Omega}$, if $\delta_{f}=\delta_{g}$, then for every $h \in \mathcal{J}_{\partial \Omega}$, we have $\delta_{f}(h)=\delta_{g}(h)$, thus $(g-f) h=h(g-f)$, this shows that $g-f \in\left\{\mathcal{J}_{\partial \Omega}\right\}^{\prime}$, the commutant of $\mathcal{J}_{\partial \Omega}$. However, it is not difficult to see that $\left\{\mathcal{J}_{\partial \Omega}\right\}^{\prime}=\mathbb{C} I$. 
Hence $g-f=\lambda I$ for some $\lambda \in \mathbb{C}$, further $Z\left(\mathcal{J}_{\partial \Omega}, \mathcal{J}_{\partial \Omega}\right) \cong \mathcal{J}_{\partial \Omega} / \mathbb{C} I$. By Proposition 3, if $\delta \in B\left(\mathcal{J}_{\partial \Omega}, \mathcal{J}_{\partial \Omega}\right)$, then $\delta$ maps $\mathcal{J}_{\partial \Omega}$ into $\mathcal{K}$. Note $\delta$ is induced by a bounded operator $T$ on $H^{2}(\partial \Omega$ ) (see [27]), that is $\delta(f)=$ $f T-T f$ for any $f \in \mathcal{J}_{\partial \Omega}$, so $T \in\left\{\mathcal{J}_{\partial \Omega}\right\}_{e}^{\prime}$, the essential commutant of $\mathcal{J}_{\partial \Omega}$. We may shows that $\left\{\mathcal{J}_{\partial \Omega}\right\}_{e}^{\prime}=\left\{T_{z_{1}}, \ldots, T_{z_{n}}\right\}_{e}^{\prime}$. In fact, it is obvious that $\left\{\mathcal{J}_{\partial \Omega}\right\}_{e}^{\prime} \subset\left\{T_{z_{1}}, \ldots, T_{z_{n}}\right\}_{e}^{\prime}$. For each $T \in\left\{T_{z_{1}}, \ldots, T_{z_{n}}\right\}_{e}^{\prime}$, if we prove that $T$ commutes essentially with $T_{\overline{z_{j}}},(j=1, \ldots, n)$, then $T$ commutes essentially with each element in $\mathcal{J}_{\partial \Omega}$ by Stone-Weierstrass theorem. Write $[T]$ as the coset of $T$ in Calkin algebra $L\left(H^{2}\right) / \mathcal{K}\left(H^{2}\right)$, then $\left[T_{z_{j}}\right]$ is a normal element in $C^{*}$-algebra $L\left(H^{2}\right) / \mathcal{K}\left(H^{2}\right)$, and $[T]\left[T_{z_{j}}\right]=\left[T_{z_{j}}\right][T]$, further $[T]\left[T_{z_{j}}^{k}\right]=\left[T_{z_{j}}^{k}\right][T]$ for each $k \geq 0$, it follows that $\exp \left(i \bar{\lambda}\left[T_{z_{j}}\right]\right)[T]=[T] \exp \left(i \bar{\lambda}\left[T_{z_{j}}\right]\right)$ for each $\lambda \in \mathbb{C}$. Thus we have

$$
[T]=\exp \left(i \bar{\lambda}\left[T_{z_{j}}\right]\right)[T] \exp \left(-i \bar{\lambda}\left[T_{z_{j}}\right]\right)
$$

Similar to the proof of Fuglede theorem (see Theorem 4.76 in [1]), for each $j(1 \leq j \leq n)$, we may set

$$
\begin{aligned}
F_{j}(\lambda) & =\exp \left(i \lambda\left[T_{z_{j}}\right]^{*}\right)[T] \exp \left(-i \lambda\left[T_{z_{j}}\right]^{*}\right) \\
& =\exp \left(i\left(\bar{\lambda}\left[T_{z_{j}}\right]+\lambda\left[T_{z_{j}}\right]^{*}\right)\right)[T] \exp \left(-i\left(\bar{\lambda}\left[T_{z_{j}}\right]+\lambda\left[T_{z_{j}}\right]^{*}\right)\right) .
\end{aligned}
$$

Since $\bar{\lambda}\left[T_{z_{j}}\right]+\lambda\left[T_{z_{j}}\right]^{*}$ is self-adjoint, it shows that $\exp \left(i\left(\bar{\lambda}\left[T_{z_{j}}\right]+\lambda\left[T_{z_{j}}\right]^{*}\right)\right)$ and $\exp \left(-i\left(\bar{\lambda}\left[T_{z_{j}}\right]+\lambda\left[T_{z_{j}}\right]^{*}\right)\right)$ are unitary elements for $\lambda \in \mathbb{C}$. Hence $F_{j}(\lambda)$ is a bounded analytic map from $\mathbb{C}$ into $L\left(H^{2}\right) / \mathcal{K}\left(H^{2}\right)$. By Liouville's theorem, we know that $F_{j}(\lambda)$ must be constant. Differentiating with respect to $\lambda$ for $F_{j}(\lambda)$ and setting $\lambda=0$ yields $\left[T_{z_{j}}\right]^{*}[T]=[T]\left[T_{z_{j}}\right]^{*}(j=1, \ldots, n)$. Since $\left[T_{z_{j}}\right]^{*}=\left[T_{\overline{z_{j}}}\right]$, we see that $T \in\left\{\mathcal{J}_{\partial \Omega}\right\}_{e}^{\prime}$. It follows that $\left\{\mathcal{J}_{\partial \Omega}\right\}_{e}^{\prime}=$ $\left\{T_{z_{1}}, \ldots, T_{z_{n}}\right\}_{e}^{\prime}$. Consequently, $B\left(\mathcal{J}_{\partial \Omega}, \mathcal{J}_{\partial \Omega}\right) \cong\left\{T_{z_{1}}, \ldots, T_{z_{n}}\right\}_{e}^{\prime} / \mathbb{C} I$, hence $H^{1}\left(\mathcal{J}_{\partial \Omega}, \mathcal{J}_{\partial \Omega}\right) \cong\left\{T_{z_{1}}, \ldots, T_{z_{n}}\right\}_{e}^{\prime} / \mathcal{J}_{\partial \Omega}$. The theorem is thus complete.

Acknowledgement. The author would be grateful to Professor Tohru Uzawa and the referee for their many invaluable suggestions.

\section{REFERENCES}

[1] R. G. Douglas, Banach algebra techniques in operator theory, Academic Press, 1972.

[2] S. Axler, J. B. Conway and G. McDonald, Toeplitz operators on Bergman spaces, Can. J. Math., XXXIV (1982), no. 2, 466-483.

[3] G. F. Cao, Composition and Toeplitz operators on general domains, Tohoku Math. J., 57 (2005), 11-22. 
[4] G. F. Cao, Cohomotopy groups and $K_{0}$-groups on connected domains, preprint.

[5] L. A. Coburn, Singular integral operators and Toeplitz operators on odd spheres, Indiana Univ. Math. J., 23 (1973), 433-439.

[6] U. Venugopalkrishna, Fredholm operators associated with strongly pseudoconvex domains, J. Funct. Anal., 9 (1972), 349-373.

[7] H. Upmeier, Toeplitz operators and index theory in several complex variables, Proceedings of Symposia in pure mathematics (Part I), 51 (1990), 585-598.

[8] N. Salinas, A. Sheu and H. Upmeier, Toeplitz operators on pseudoconvex domains and foliation $C^{*}$-algebras, Ann. of Math., 120 (1989), 531-565.

[9] N. P. Jewell and S. G. Krantz, Toeplitz operators and related function algebras on certain pseudoconvex domains, Trans. Amer. Math. Soc., 252 (1979), 297-312.

[10] I. Raeburn, On Toeplitz operators associated with strongly pseudoconvex domains, Studia Math., 63 (1979), 253-258.

[11] N. P. Jewell, Fredholm Toeplitz operators on strongly pseudoconvex domains, Studia Math., T.LXVIII (1980), 25-34.

[12] J. Janas, Toeplitz operators related to certain domains in $\mathbb{C}^{n}$, Studia Math., T.LIV (1975), 73-79.

[13] H. Sato and K. Yabuta, Toeplitz operators on strongly pseudoconvex domains in Stein spaces, Tohoku Math. J., 30 (1978), 153-162.

[14] K. Yabuta, A remark to a paper of Janas "Toeplitz operators related to certain domains in $\mathbb{C}^{n}$ ", Studia Math., T.LXII (1978), 73-74.

[15] L. Boutet de Monvel and V. Guillemin, The spectral theory of Toeplitz operators, Princeton U. Press, Annals of Math. series no. 99, 1981.

[16] V. Guillemin, Toeplitz operators in n-dimensions, Integral Equations and Operator Theory, 7 (1984), 145-205.

[17] L. Boutet de Monvel, On the index of Toeplitz operators of several complex variables, Inventiones Math., 50 (1979), 249-272.

[18] S. G. Krantz, Function theory of several complex variables, Tohn Wiley \& Sons, 1982.

[19] R. E. Curto, Fredholm and invertible tuples of bounded linear operators, Trans. Amer. Math. Soc., 266 (1981), 129-159.

[20] G. F. Cao and Ch. Y. Zhong, Automorphism groups of Toeplitz algebras on certain pseudoconvex domains, Northeast. Math. J., 14 (1998), no. 1, 112-118.

[21] J. Dugundji, Topology, Allyn and Bacon, Inc., Boston, 1966.

[22] M. Rørdam, F. Larsen and N. Laustsen, An introduction to $K$-theory for $C^{*}$-algebras, Cambridge Univ. Press, 2000.

[23] R. G. Douglas, $C^{*}$-algebra extensions and $K$-homology, Princeton University Press and University of Tokyo Press, 1980.

[24] S. Sakai, On a problem of Kaplansky, Tohoku Math. J., 12 (1960), 31-33.

[25] R. V. Kadison, Notes on the Gelfand-Neumark theorem, Contemporary Math., 167 (1994), 21-53.

[26] A. Ya. Helemskii, The homology of Banach and topological algebras, Kluwer Academic Publishers, 1989.

[27] R. V. Kadison, Derivations of operator algebras, Ann. of Math., 83 (1966), 280-293. 
Department of Mathematics

Guangzhou University

Guangzhou 510006

China

guangfucao@163.com 